\title{
Implementation of Knowledge Management in Construction Projects
}

\author{
Kasim A Korkmaz* and Saeed Bahidrah \\ School of Visual and Built Environments, Eastern Michigan University, USA \\ *Corresponding author: Kasim A Korkmaz, School of Visual and Built Environments, Eastern Michigan University, Ypsilanti, Michigan, USA
}

\begin{abstract}
Knowledge management is a tool that can be used by construction companies at management level to reduce time consumption on data process and transmittal in various stages of existing and future projects. This tool helps in communication between different layers of the companies and in transferring data and information from the completed projects in the past. Since the construction industry is one of the biggest industries in the world, it has lots of different applications and practices with diverse projects. Based on a comprehensive literature review of empirical research and detailed case studies, it is found that, there have been general, as well as project specific elements existing to prevent firms from applying knowledge acquired from their past performance. Lack of coordination and communication slows down the transfer of data from past to present and future projects. Complexity of projects constitutes the main obstacle blocking firms from utilizing knowledge in new settings. Further, the study proposes several strategies for effective utilization of knowledge management in existing and future projects to maximize the efficiency and effectiveness of knowledge management use in construction projects resulting in reduction of time and cost and an increase in quality.
\end{abstract}

Keywords: Knowledge management; Construction industry; Communication; Data transfer; Recommendations to use of knowledge management

\section{Introduction}

In the Construction industry, it is critical to transfer data and information from projects completed in the past. However, there is still a lack of systematic approaches in place to learn from previous projects in many ways [1]. Valuable data collected from small, medium and large residential and commercial projects are rarely used to make decisions on similar projects firms undertake in their future work. The industry is still dominated by non-systematic individualized idiosyncratic approaches relying on extensive personal experience and attitudes [2]. Construction professionals mostly give their decisions based on time, cost and quality associated with a given project depending on their judicious judgement. There is no frequent practice for using previous knowledge from past projects. The manufacturing and service industries have long applied the concept of knowledge management to maximize efforts of increasing the efficiency and effectiveness of their projects. Empirical, as well as case studies have concluded that knowledge management reduces time span of projects, diminishes the costs associated with the undertaking of projects and increases the quality, as well as sustainability of projects.

Knowledge management is defined as identification, optimization and active management of intellectual assets to create value, increase productivity, and gain and sustain competitive advantage [3]. Knowledge management is a multidimensional construct composed of three principal areas. First, stakeholders in any project must identify the essential information to be shared and utilized for the goodness of the project. Second, the information identified should be organized, analyzed, presented and disseminated in the optimal manner where all stakeholders can easily understand and utilize the output, knowledge, produced by the process. Third, stakeholders must retain, refine and validate the information obtained from projects to be used in future endeavors. This information does not only include raw numbers collected from a specific project, timelines, projected costs and standards/ benchmarks, but also the rich expertise offered by project staff and stakeholders in dealing with the challenges, problems, opportunities and completion of projects.

This study highlights the main barriers standing between knowledge management and its implementation in the construction industry. The research also proposes a framework for optimal utilization of knowledge management in construction projects reducing time and cost while increasing quality and sustainability. The study proposes four stages in the road for construction firms and professionals to maximize the use of knowledge management in projects (Figure 1). Functions of knowledge management systems is given in Figure 2 [4]. Based on this approach, there are four steps for implementation. first, construction firms and professionals must increase awareness of the importance and significance of knowledge management to stakeholders. Second, since every project is unique in the industry, an identification of crucial data 
and knowledge components must be specified prior to the project implementation phase. Third, construction firms must invest in efficient technologies to retain, share and validate the information obtained from projects.

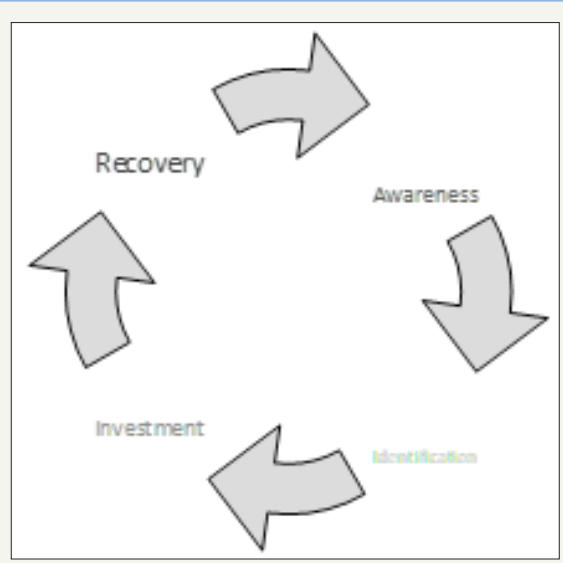

Figure 1: Four stages of knowledge management.

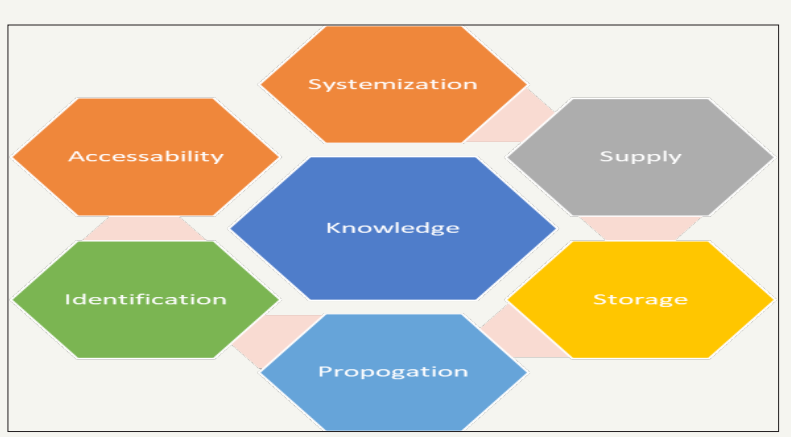

Figure 2: Functions of knowledge management systems [4].

Finally, construction firms must recover all relevant knowledge before the commencement of new projects to better estimate the time, costs, quality and sustainability of projects. Using this framework, construction firms are expected to reduce time, costs, delays and increase quality, efficiency and sustainability. Knowledge management has infiltrated the world of managing and organizing organizational activities [5]. Construction organizations have utilized the project management tools in project planning and execution to achieve project management performance outcomes in terms of time, cost and quality [6,7]. As the construction industry uses a considerable number of knowledge workers, implementing Knowledge Management for project planning and execution is the key to sustaining the growth of construction organizations and industry, particularly when Knowledge Management implementation is linked to project performance outcomes. Gunasekera et al. [8] have reviewed the knowledge management processes, knowledge conversion modes and critical success factors.

\section{Barriers in implementation of knowledge management}

In construction projects, there are various barriers in implementation of knowledge management as given in Figure 3. The lack of coordination among construction professionals during a project is the main reason behind the limited application of knowledge management within the industry [9]. On-site workers do not communicate well with off-site staff in many situations resulting in delays, higher costs and lower quality. Infrequent meetings between construction staff, stakeholders and suppliers reduces the efficiency in implementing best practices standards in completing projects. The proliferation of virtual teams in construction projects also lowers face-to-face interactions among staff, which is likely decreasing levels of coordination among various parties to the project. Further, outsourcing and subcontracting decentralizes the tasks associated with the project making it difficult on the various stakeholders to coordinate meetings, share information and utilize collective or even individual expertise.

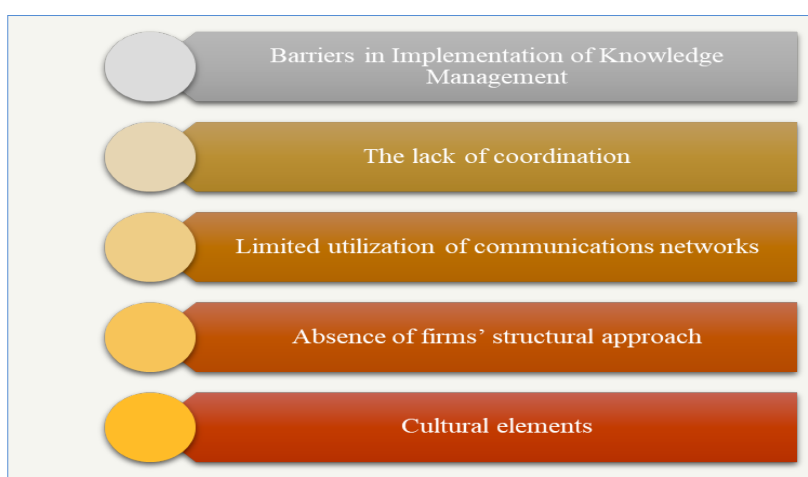

Figure 3: Barriers in implementation of knowledge management.

Second reason impeding the efficient implementation of knowledge management in the construction industry is the limited utilization of communications networks. Despite, the proliferation of advanced applications for workers' communications in real time, many construction projects still do not heavily rely on this form of communications. Further, many small and medium size construction firms do not effectively communicate the detailed books of previous projects adding time, effort and cost to doing an already conducted research. This failure of communications slows down the project and does not effectively create a knowledge base that is shared by projects' staff overtime [10].

Third, the absence of firms' efforts and desire to generate a useful body of knowledge that can be transmitted into other projects is a significant hindrance to the successful implementation of knowledge management in the construction industry. Construction projects are highly idiosyncratic requiring unique processes and procedures that are difficult to generalize as a usable collective body of knowledge [1]. This leads stakeholders, experts and professionals to develop an attitude of "every project is unique, thus there is no point of building a knowledge base." While unique elements exist in every project, they do in every production, manufacturing and service protocol and this has not prevented. Fourth, empirical investigations and detailed case studies in the construction management field have noted to the lack of education, awareness, training and implementation of knowledge management among construction professionals. Construction workers, site engineers, managers, clients and the administrative apparatus in many cases do not know the potential benefits gained from knowledge 
management. They also are not aware of the costs, efforts and time that is saved simply by applying knowledge management processes and methods.

Finally, cultural elements prevent the construction industry from the effective implementation of knowledge management [11]. The construction industry is growing at an exponential rate in developing countries such as Brazil, Turkey, India and the Middle East. The industries in such countries lack the technological sophistication, men powers' skillsets and maturity compared to the markets of the United States, United Kingdom, Japan and Germany. Many professionals working in those markets are still skeptical about the usefulness and benefits of knowledge management. They favor the individual unique approach to the utilization of knowledge based on experience, merit and good judgement.

\section{Implementation of knowledge management in construction}

Knowledge is a quantity which had a multitude of components attached to it. The concepts associated with knowledge. They usually are centered around the traditionally definition of the set of "facts, information, and skills acquired through experience or education; the theoretical or practical understanding of a subject or the sum of what is known as information held on a computer system; awareness or familiarity gained by experience of a fact or situation $[2,12,13]$. A distinction between the different classes of knowledge at play can be precisely defined and categorized into three main knowledge types as they relate to the construction industry: domain knowledge, organizational knowledge and project knowledge [9]. Domain knowledge includes administrative information, including local laws, zoning regulations, permits and regulations, standards and the maintenance of an adequate product databases, for example. This type of information is meant to be available to all companies who operate in a common business environment. This knowledge, stored in a wide variety of ways which range from the systematic, highly organized electronic storage techniques to human storage as well as cultural transmission, represents all that there is to be known about a particular exterior environment in which a particular construction company happens to operate at the moment. Issues associated with such knowledge are often relatively minor when compared to those which arise when considering the knowledge processes at next step. Issues may arise in terms of integration and operations under a multitude of concurrent set of domain knowledge requirements, such as when operating abroad under different local rules yet abiding to the laws of the home nation.

Another important aspect of the knowledge process as it is relevant to the construction industry is the organizational knowledge of the company. Such knowledge is most often company specific and represents both an intellectual and financial capital for the firm. It is often stored in the company records formally, and informally in the day-to-day skilled processes that make up the operational structure of the firm. Organizational knowledge expresses the competence of the firm in bringing together and integrating the personal talents, skills or special experience and competence of the staff. Moreover, it is a somewhat direct measure of the efficiency of the firm's cross-organizational knowledge as it is set-up to establish and protect business relationships with contractors, architects, and engineering.

The third axis of the knowledge concept is the more specific, one-off set of knowledge which is required per the specific nature of a specific project. Thus, project knowledge represents "the potential for usable knowledge and is at the source of much of the knowledge on which these firms depend" [1]. A competent project knowledge process should be capable of not only managing the trove of data and information generated by the project, but also the less constrained knowledge that is created by the interaction between the various firms and professional entities involved. Therefore, this valuable information such as what were the optimal solutions to technical problems, or how mistakes were avoided or dealt with in the past, the multitude of company partnerships that arise during each project are generally not able to capitalize on this potential for creating and more efficiently using knowledge. Specific problems in this area include the formalized recording, or general lack thereof, of project records and the often-unrecorded memory of processes, problems and related solutions. The fourth type of domain knowledge involves that relevant to the indicators of the performance and efficiency of the operations of the company themselves. This knowledge is crucial to understand correctly to be able to assess the state of progress in the other domains. In a DKM Economic Consultants report, major construction industry indicators are included "Gross fixed capital formation, construction employment, construction earnings, wholesale prices for construction materials, construction cost, price inflation, planning permissions for constructional development, registrations, house price and construction confidence [14].

\section{Establishing a knowledge sharing culture - potential solutions}

Due to the complexity of most construction projects, from design to inception and operational review, these three knowledge categories are often inter-linked and integrated into information layers which often affect each other, especially the more so in recent years with the widespread emergence and use of the Internet and communication medias. While it is certainly true that knowledge involves the manipulation of data from the point of view of experience, the fact remains that what is precisely meant by knowledge must involve the even more abstract notion of tacit knowledge. Within the last twenty years, there has been an emerging interest in the tacit dimension of knowledge, which is perhaps the hardest component to manage.

Individual knowledge is also an important aspect of the successful implementation of the principles of Knowledge Management into the day-to-day practices of the construction industry. Employees should be selected in terms of self-starting abilities and the seeking out of opportunities and design in their own work under little direction while their awareness of Knowledge 
Management can be improved by using training and enhancing their participation in education, conferences and similar knowledgesharing activities. This will allow employees the opportunity to reflect upon their work, trade stories and ideas with co-workers, or catch up on professional theory and practice [15].

In the context of Project Knowledge solutions for capturing and sharing project knowledge, to reduce the required amount of reinventing the wheel, include site visits, audits, discussion and informal meetings followed by their documentation [9]. In attempting to manage organizational knowledge within construction organizations, one must worry about product, or the technical knowledge; process, representing the procedural and regulatory knowledge; and people, which identifies and connects specific skills with experiences [16]. Detailed components of such solutions include knowledge dissemination workshops; knowledge exchange seminars and departmental meetings; summary reports and project award scheme [16].

\section{Decision making in construction projects}

The Knowledge Management is a tool that helps in decision making as well. Knowledge Management has started to evolve into a mature reality and that today many worldwide industries, organizations and various institutions are increasingly understanding and using the tools offered by Knowledge Management. In fact, the global Knowledge Management market expanded to the record amount of $\$ 8.8$ billion in 2005 while applying Knowledge Management techniques also helped save \$31 Billion in annual re-invention costs at Fortune 500 companies in that same year. The broader application context of Knowledge Management, including learning and educational opportunities, offers similarly powerful forecasts [17]. Therefore, the inertia has slowly built up and that the tide is now slowing stacking up on the side of a near future when serious implementation of the tenets of Knowledge Management will allow true progress and advances in the way that the overall informational content is managed by construction industries, both in a manner directly relevant to the project as well as, equally importantly, that pertaining to the domain and organizational aspects of knowledge dynamics.

\section{Integration of knowledge management in the processes}

Organizations manage knowledge in an intentional and directed manner. In recent years, a more intentional approach to organizational knowledge management has interested both scholars and the industry, leading to numerous approaches on knowledge management currently being analyzed, studied and adopted and used by organizations, each having its own qualities, requirements, strengths, weaknesses, benefits and potentials. The first approach is generally IT dependent, and tracks knowledge management and its spread and efficiency throughout the company. This method is generally technologies centered and the necessary requirements for an IT system must render it sufficiently capable of being a powerful tool for increasing the efficiency of
Knowledge Management, for example by supporting the entire lifecycle of the Knowledge Management process. The IT system must also be a good source of performance indicators and other validating mechanisms, while its flexibility, ease of use and being able to be integrated with other systems is also a must [9]. This type of knowledge management leverages the informational content of various communication technologies to unify the organization's knowledge assets. Secondly, another approach tracks people's individual knowledge management and focuses on the organizational behavioral approach. Those two knowledge management approaches underline the current distinction which is made between process-centered and product-centered approaches. The leadership in the construction can influence the use of shared knowledge between the senior and junior project personnel, which subsequently affects the degree of success of project execution [18]. Construction projects encounter many challenges throughout the project life cycle which affect the morale of project team members, top management plays an important role in proper delegation of responsibilities and tasks at different levels [19].

The process-centered approach looks at knowledge management from a social communication point of view where knowledge is closely tied to the person who developed it and is shared mainly through person-to-person contact. Product-centered methods, on the other hand, focuses on documentary knowledge and the storage and reuse of computer based corporate memories. It is also referred to as "content-centered" approach." A complementary approach whose design and implementation directly results from applying the principles of Knowledge Management in the specific social-technical environment of the construction industry is the Accident Root Cause Tracing Method. This procedure is established in both the operational and project knowledge requirements and its philosophy, besides the logical immediate training considerations, depends on management procedures which should be designed to identify and remove unsafe conditions in a proactive manner [20].

Knowledge management covers two main parts as knowledge representation and knowledge interoperability. These parts have an integration over the process representation, and process exploration. In construction projects, process representation and process exploration are the main crucial items that leads the communication process in construction. In the architecture of knowledge management tools for construction projects, storage, access control, for various data/information security, interface communication, interoperability, service and presentation will be the main layers to carry out with the development of the knowledge management model. Flow charts for Knowledge management model in an integrated approach is given in Figure 4.

A. Storage: An online repository is used for data storage. The back-end database will be accessed using regular SQL queries.

B. Access: Access control, for data/information security, is provided through authentication and authorization. 
C. Communication: Interface communication is provided through the Internet. An end-user or application can communicate within the portal using the following data formats: OWL files, Extensive Markup Language (XML) and process-structure and Microsoft Project data files, for importing/exporting schedule information.

D. Interoperability: It is a semantic representation.
Interoperability of different data/ information/ knowledge is then achieved.

E. Service: A set of core services is available to portal users: building and editing, merging, process structure building and editing, and process-structure merging. Similar to the earlier point, the service will be based on well-established Web Services standards.

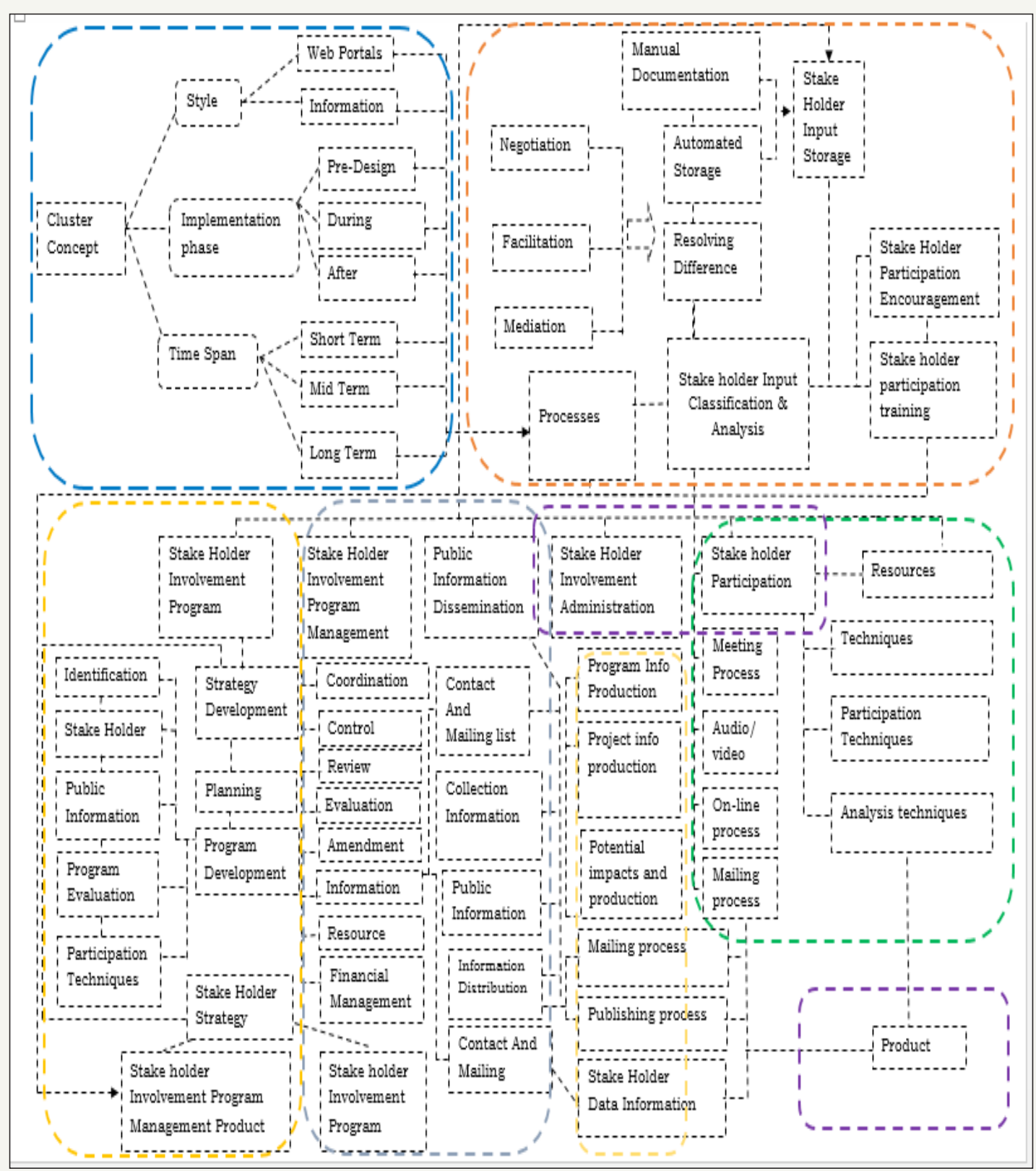

Figure 4: Integrated knowledge management model.

\section{Conclusion}

Activities in Knowledge Management in general, and in the construction industry in particular, are still today all over the map and without a clear guiding light, even though the monumental increases in research and spending in this domain are not to be discarded. The current climate in the general construction industry environment is one of relative waiting and looking to see which opportunity present themselves with regards to revamping their knowledge and communication systems from the ground up in a way which incorporates the findings associated with better incorporating the academic understanding presented above as solution to the problems arising in construction projects. Figure 5 summarizes the mechanism of the knowledge in construction. 


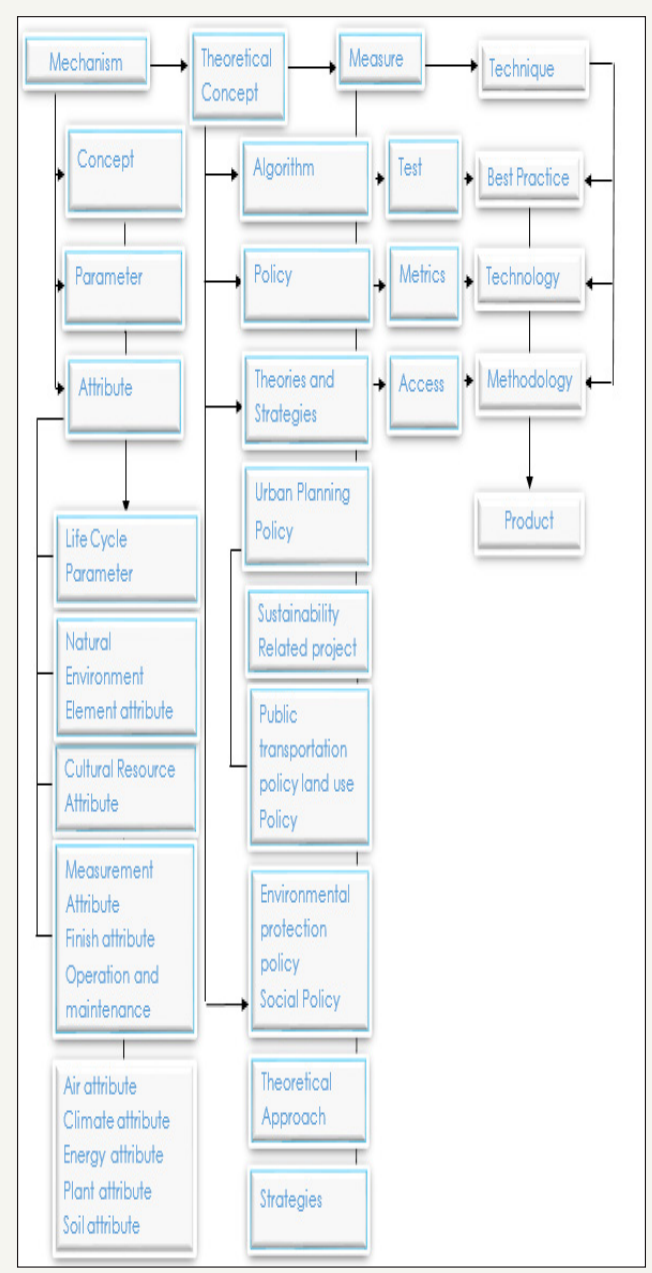

Figure 5: Mechanism of knowledge in construction.

The elevated focus on just the IT aspect of the issue has be noted, and it is even the case that research has even shown that there existed, in some case, inverse correlations between IT investments and business performance [17]. The clear difference between information-processing capabilities offered using the latest technologies versus the very different sense-making capabilities, as well as the mechanistic emphasis of technology-based linkages can thus be contrasted with the organic and hard to measure and define nature of the required relationships needed for nurturing knowledge processes. It is obvious from the data researched at this point that the construction industry at large needs to better manage its knowledge-based and informational resources to improve business processes, streamline operations, increase overall efficiency and satisfy its clients. Several issues of particular relevance which need to be addresses in priority are, first of all, the recognition of the need to address Knowledge Management in a comprehensive, industry-wide manner; the formulation of viable Knowledge Management strategies which are proactive and aimed at performance, as well as a need to understand the overall impact that establish such new procedures would have on the organizational structure and working practices of a typical construction company [2].

\section{References}

1. Lima C, Rezgui Y, Wetherill M, Zarli A (2002) Knowledge management for the construction industry: the e-cognos project. Itcon 7: 183-195.

2. Carrillo PM, Anumba CJ, Kamara JM (2000) Knowledge management strategy for construction: key I.T. and contextual issues.

3. Kanapeckiene L, Naimaviciene J, Tupenaite L (2008) Knowledge management model for construction projects. $8^{\text {th }}$ International Conference: Reliability and Statistics in Transportation and Communication.

4. Tupenaite L, Kanapeckiene L, Naimaviciene J (2008) Knowledge management model for construction projects. The $8^{\text {th }}$ International Conference "Reliability and Statistics in Transportation and Communication, 15-18 October 2008, Riga, Latvia.

5. Heisig PS, Surai OA, Kianto A, Kemboi C (2016) Knowledge management and business performance: global experts' views on future research needs. Journal of Knowledge Management 20(6): 1169-1198.

6. Handzic M, Durmic N (2015) Knowledge management, intellectual capital and project management: connecting the dots. The Electronic Journal of Knowledge Management 13(1): 51-61.

7. Zhai X, Liu AMM, Fellows R (2014) Role of human resource practices in enhancing organizational learning in Chinese construction organizations. Journal of Management in Engineering 30(2): 194-204.

8. Gunasekera VS, Chong SC (2018) Knowledge management for construction organizations: a research agenda. Kybernetes. 
9. Mohammed AH, Wei LL (2005) The development of a knowledge sharing culture in the construction industry. Intra-departmental paper, Department of Property Management.

10. Finegan AD, Maqsood T, Walker DHT (2003) Sustainability and innovation in management and technology: a soft approach to solving hard problems in construction project management. Second International Conference on Construction in the $21^{\text {st }}$ Century (CITC-II).

11. Levitt RE, Scott WR, Will AJ (2007) Understanding knowledge acquisition, integration and transfer by global development, engineering and construction firms. CRGP Working Paper Series No. 28.

12. Chaminda PC, Amaratunga D, Haigh R (2002) Managing the construction worker in a knowledge environment. Intra-departmental paper Research Institute for the Built and Human Environment, The University of Salford, Salford M5 4WT, United Kingdom.

13. Bosch SP, Postma T (2006) Knowledge transfer in project-based environments: a study on innovation projects in the construction industry. OLKC 2006 conference at the University of Warwick, Coventry.

14. DKM Economic Consultants: Construction industry indicators. (2005) Construction Policy and NDP Coordination Unit of the Department of the Environment, Heritage and Local Government, Dublin, Ireland, by DKM Economic Consultants Ltd. 3
15. Grisham T, Maqsood T, Srinivasan P, Walker DHT (2006) Frameworks for knowledge management initiatives in the field of project management using metaphor for improved visibility. Joint International Conference on Construction Culture, Innovation, and Management (CCIM).

16. Egbu CO, Robinson HS (2005) Construction as a knowledge-based industry in knowledge management in construction. In: Anumba CJ, Egbu CO, Carrillo P (Eds.), Management of knowledge in project environments, Blackwell Publishing, UK.

17. Yogesh MY (2005) Integrating knowledge management technologies in organizational business processes. Journal of Knowledge Management $9(1)$.

18. Zizek S, Mulej M, Cic Z (2017) Results of socially responsible transformational leadership: increased holism and success. Kybernetes 46(3): 400-418.

19. Aleksic A Puskaric H, Tadic D, Stefanovic M (2017) Project management issues: vulnerability management assessment. Kybernetes 46(7): 11711188

20. Abdelhamid TS, Everett JG (2000) Identifying root causes of construction accidents. ASCE Journal of Construction Engineering.
Creative Commons Attribution 4.0

International License

For possible submissions Click Here

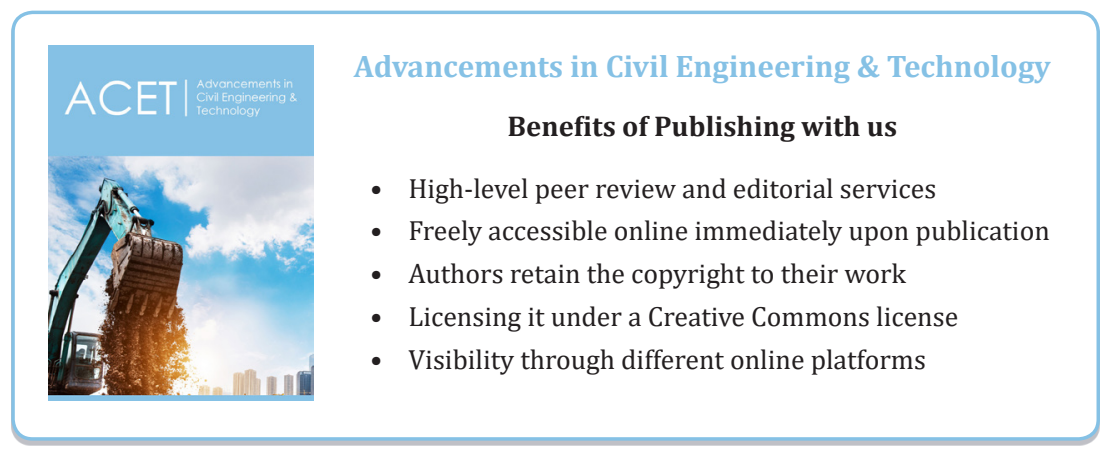

